\title{
EFICIÊNCIA DO POLITUNGSTATO DE SÓDIO NO FRACIONAMENTO DENSIMÉTRICO DA MATÉRIA ORGÂNICA DO SOLO(1)
}

\author{
Paulo Cesar Conceição ${ }^{(2)}$, Madalena Boeni ${ }^{(3)}$, Jeferson Dieckow ${ }^{(4)}$, \\ Cimélio Bayer ${ }^{(5)}$, Ladislau Martin-Neto ${ }^{(6)}$ \& João Mielniczuk ${ }^{(5)}$
}

\begin{abstract}
RESUMO
Soluções de iodeto de sódio (NaI) e de politungstato de sódio (PTS) têm sido utilizadas, de forma indistinta, no fracionamento densimétrico da matéria orgânica (MO) do solo. O objetivo deste estudo foi avaliar o efeito desses dois produtos em soluções de mesma densidade $\left(1,80 \mathrm{~kg} \mathrm{~L}^{-1}\right)$, bem como do aumento da densidade da solução de PTS (2,0 e 2,2 $\left.\mathrm{kg} \mathrm{L}^{-1}\right)$, na obtenção de C na fração leve (FL) da MO do solo. As características estruturais da FL obtida com as diferentes soluções também foram avaliadas por ressonância magnética nuclear do ${ }^{13} \mathrm{C}\left(\mathrm{RMN}-{ }^{13} \mathrm{C}\right)$ neste estudo, o qual foi realizado com amostras da camada de 0-5 cm de dois solos brasileiros (PVd-Argissolo Vermelho e LVd-Latossolo Vermelho). A dispersão do solo foi realizada com ultra-som ( $250 \mathrm{~J} \mathrm{~mL}^{-1}$ no PVd e $450 \mathrm{~J} \mathrm{~mL}^{-1}$ no LVd); a suspensão de $20 \mathrm{~g}$ de solo e $80 \mathrm{~mL}^{-1}$ de solução foi centrifugada $(2.000 \mathrm{~g}, 90 \mathrm{~min})$, e a FL, obtida pela filtragem do sobrenadante em filtro de fibra de vidro, sendo o $\mathrm{C}$ desta fração analisado por combustão seca (Shimadzu TOC-V CSH). O uso da solução de PTS aumentou a recuperação de C da FL em 152 \% no PVd e em 166 \% no LVd, em comparação à solução de $\mathrm{NaI}$ de mesma densidade $\left(1,8 \mathrm{~kg} \mathrm{~L}^{-1}\right)$. $\mathrm{O}$ incremento da densidade da solução de PTS também aumentou a obtenção de C na FL, o qual foi mais expressivo na densidade de 1,8 para $2,0 \mathrm{~kg} \mathrm{~L}^{-1}(57 \% \mathrm{em}$ ambos os solos) do
\end{abstract}

\footnotetext{
(1) Parte da Tese de Doutorado do primeiro autor submetida ao Programa de Pós-graduação em Ciência do Solo (PPGCS), Universidade Federal do Rio Grande do Sul - UFRGS. Recebido para publicação em novembro de 2006 e aprovado em julho de 2007.

(2) Pós-Doutorando do Departamento de Solos, Universidade Federal do Rio Grande do Sul - UfRGS. Caixa Postal 15100, CEP 91501-970 Porto Alegre (RS). Bolsista do CNPq. E-mail: pcconceicao@hotmail.com

(3) Doutoranda do PPGCS, UFRGS. E-mail: madaboeni@hotmail.com

(4) Professor do Departamento de Solos e Engenharia Agrícola, Universidade Federal do Paraná - UFPR. CEP 80035-050 Curitiba (PR). E-mail: jefersondieckow@ufpr.br

(5) Professor do Departamento de Solos, UFRGS. Bolsista do CNPq. E-mail: cimelio.bayer@ufrgs.br

(6) Pesquisador da Embrapa Instrumentação Agropecuária. Caixa Postal 741, CEP 13560-970 São Carlos (SP). Bolsista do CNPq. E-mail:martin@cnpdia.embrapa.br
} 
que de 2,0 para 2,2 $\mathrm{kg} \mathrm{L}^{-1}$ (21 \% no PVd e 5 \% no LVd). Com base nos teores de Fe e C da fração argila, estimou-se que a contaminação da FL com C da fração argila, quando do uso das soluções de PTS, variou de 1,9 a 3,5 \% no LVd e de 6,8 a $10,4 \%$ no $\mathrm{PVd}$, os quais foram considerados baixos perante o alto percentual de incremento na recuperação de $\mathrm{C}$ na $\mathrm{FL}$. $\mathrm{O}$ incremento da razão $\mathrm{C}$ Alquil/C-O-alquil e do $\mathrm{C}$ carbonila na análise de $\mathrm{RMN}-{ }^{13} \mathrm{C}$ sugere incremento na recuperação de $\mathrm{FL}$ em estádios mais avançados de decomposição com o uso de PTS e incremento da densidade da solução, tendo sido esses resultados mais expressivos no LVd. Com base no incremento da obtenção de $C$ e na baixa contaminação da FL com $C$ da fração argila, recomenda-se o uso da solução de PTS $2,0 \mathrm{~kg} \mathrm{~L}^{-1} \mathrm{em}$ estudos de fracionamento densimétrico da MO do solo.

Termos de indexação: fracionamento densimétrico, iodeto de sódio, matéria orgânica particulada.

\title{
SUMMARY: EFFICIENCY OF SODIUM POLYTUNGSTATE IN DENSITY FRACTIONATION OF SOIL ORGANIC MATTER
}

\begin{abstract}
Sodium iodide (NaI) and sodium polytungstate (SPT) solutions are being indistinctively used in density fractionation procedures of soil organic matter (SOM). This study aimed to evaluate these two solutions, adjusted to a density of $1.8 \mathrm{~kg} \mathrm{~L}^{-1}$ (NaI-1.8 and SPT-1.8), and particularly of SPT adjusted to densities of 2.0 (SPT-2.0) and $2.2 \mathrm{~kg} \mathrm{~L}^{-1}$ (SPT-2.2), with regard to their capacity of recovering the light fraction (LF) of SOM. A $20 \mathrm{~g}$ soil sample of the $0-5 \mathrm{~cm}$ layer of an Acrisol (PVd) and of a Ferralsol ( $L V d)$ was dispersed by sonication at $250 \mathrm{~J} \mathrm{~mL}^{-1}$ and $450 \mathrm{~J} \mathrm{~mL}^{-1}$, respectively for each soil, in $80 \mathrm{~mL} \mathrm{NaI}$ or SPT solution. After centrifugation $(2.000 \mathrm{~g}, 90 \mathrm{~min})$, the $L F$ in the supernatant was recovered on a fiberglass filter, dried and analyzed for $C$ content determination (TOC dry combustion) and chemical structure characterization $\left({ }^{13} \mathrm{C}\right.$ nuclear magnetic resonance spectroscopy). The SPT-1.8 increased LF-C recovery by $152 \%$ in the PVd and $166 \%$ in the LVd, compared to NaI-1.8. Besides, the higher SPT-2.0 density increased LF-C recovery by $57 \%$ in both soils as compared to SPT-1.8. Furthermore, SPT-2.2 recovered $21 \%$ and $5 \%$ more LF-C than SPT-2.0, in the PVd and LVd, respectively, but this additional recovery was not as pronounced as when density was changed from 1.8 to $2.0 \mathrm{~kg} \mathrm{L^{-1 }}$. Based on data of Fe content in $L F$ and $C$ and $F e$ content in clay fraction, we estimated that the contamination of clay- $C$ in the LF using SPT solutions varied 1.9-3.5 \% in the LVd and 6.8-10.4\% in the $P V d$. Theses values were considered low compared to the high increments obtained in $L F$ $C$ recovery. The increases in both alkyl-C/O-alkyl-C ratio and carbonyl-C abundance when NaI was substituted by SPT and when SPT densities increased, suggested that more decomposed LF material was recovered, which was more evident in the LVd. Based on our results, we suggest using SPT solution adjusted to a density of $2.0 \mathrm{~kg} \mathrm{~L} \mathrm{~L}^{-1}$ in studies focusing on densimetric fractionation of SOM.
\end{abstract}

Index terms: densimetric fractionation, sodium iodide, particulate organic matter.

\section{INTRODUÇÃO}

Na ciência do solo tem sido expressivo o interesse no entendimento do efeito de sistemas de uso e manejo na dinâmica e qualidade da matéria orgânica (MO), cujo avanço, em grande parte, está relacionado ao uso de técnicas de fracionamento físico granulométrico e densimétrico da MO, as quais permitem a quantificação e caracterização de diferentes compartimentos do C no solo (Christensen, 1992; Cambardella \& Elliott, 1992; Golchin et al., 1994a,b; Bayer et al., 2001; Freixo et al., 2002a,b,c; Diekow et al., 2005).
O fracionamento densimétrico permite a obtenção de uma fração orgânica particulada (fração leve-FL), derivada dos resíduos de plantas, raízes e hifas, que ainda apresentam estruturas celulares reconhecíveis, e de uma fração orgânica associada aos minerais (fração pesada-FP). A FL é mais sensível às práticas de manejo, o que a torna uma importante fração na avaliação da qualidade do sistema de manejo no curto prazo (Bayer et al., 2001; Conceição et al., 2005). A FP representa a MO do solo em estádio avançado de decomposição, sendo mais estável e com maior tempo de residência no solo do que a FL (Christensen, 2001), 
cujo estoque de $\mathrm{C}$ é altamente dependente da textura e mineralogia do solo (Hassink et al., 1993; Feller \& Beare, 1997).

As densidades das soluções utilizadas no fracionamento densimétrico da $\mathrm{MO}$ variam de 1,0 a $2,4 \mathrm{~kg} \mathrm{~L}^{-1}$, sendo o iodeto de sódio $(\mathrm{NaI})$ e o politungstato de sódio (PTS) usados preferencialmente a soluções orgânicas, que apresentam elevada toxidez (Christensen, 1992). Normalmente, as soluções de $\mathrm{NaI}$ e de PTS são utilizadas de forma indistinta, não tendo sido avaliada a eficiência relativa desses produtos no rendimento da FL. Entretanto, Shang \& Tiessen (2001) comentam brevemente sobre a baixa recuperação de $\mathrm{C}$ na FL quando do uso de solução de $\mathrm{NaI} 1,7 \mathrm{~kg} \mathrm{~L}{ }^{-1}$, em comparação à solução de PTS de mesma densidade, não tendo sido identificada a razão disso.

Outro aspecto a ser considerado é a influência da densidade da solução na recuperação da FL da MO do solo. Shang \& Tiessen (2001), utilizando solução de PTS nas densidades de 1,8, 2,0, 2,2 e 2,4 $\mathrm{kg} \mathrm{L}^{-1}$ para quantificar a FL na fração silte de um Latossolo tropical (190 $\mathrm{g} \mathrm{kg}^{-1}$ de argila), encontraram incremento do rendimento de $\mathrm{C}$ da FL variando de 39 a $163 \%$, pela utilização das soluções mais densas, em comparação à solução com $1,8 \mathrm{~kg} \mathrm{~L}^{-1}$. Sohi et al. (2001) não obtiveram aumento na recuperação da FL livre (interagregados) pela alteração da densidade da solução de $\mathrm{NaI}$ de 1,6 para $1,8 \mathrm{~kg} \mathrm{~L}^{-1}$. Entretanto, a elevação da densidade da solução aumentou a recuperação da FL oclusa (intra-agregados), indicando que esse efeito pode ser mais efetivo em frações orgânicas em estádio mais avançado de decomposição (Golchin et al., 1994a; Sohi et al., 2001). A distinção entre as soluções de diferentes produtos e densidades na capacidade de separação de FL com estádios diferenciados de decomposição pode ser avaliada a partir da utilização de técnicas espectroscópicas, com destaque para a ressonância magnética nuclear do ${ }^{13} \mathrm{C}\left(\mathrm{RMN}-{ }^{13} \mathrm{C}\right)$, a qual já vem sendo amplamente empregada em estudos da caracterização da MO do solo no Brasil (Bayer et al., 2000; Roscoe et al., 2004; Dick et al., 2005).

Os resultados referentes à comparação da eficiência de soluções de NaI e de PTS e da variação da densidade da solução no rendimento de C na FL na literatura são escassos, mas sugerem que estes produtos não devem ser utilizados de forma indistinta e que há portanto, necessidade de estudos visando à padronização de um método que permita a comparação de resultados obtidos por diferentes grupos de pesquisa, o que representaria grande avanço no entendimento da dinâmica da MO em diferentes solos e condições ambientais. Nesse sentido, realizou-se o presente estudo, o qual teve por objetivo avaliar a eficiência das soluções de NaI e de PTS, e de diferentes densidades do PTS, no rendimento de $\mathrm{C}$ da FL da MO em dois solos brasileiros com textura e mineralogia distintas.

\section{MATERIAL E MÉTODOS}

Amostras da camada de 0-5 $\mathrm{cm}$ de um Argissolo Vermelho distrófico-PVd e de um Latossolo Vermelho distroférrico-LVd (Embrapa, 1999) foram utilizadas no presente estudo. Em ambos os solos, as amostras foram coletadas em experimento de longa duração, em tratamentos com alta adição anual de resíduos vegetais e sem revolvimento do solo. No Argissolo, foram coletadas amostras de solo sob plantio direto por 18 anos na sucessão aveia (Avena strigosa) + ervilhaca (Vicia sativa)/milho (Zea mays) + caupi (Vigna unguiculata), enquanto no Latossolo a área amostrada vinha sendo mantida sob pastagem permanente de braquiária (Brachiaria decumbens) por nove anos. A distribuição granulométrica e a caracterização da fração argila dos solos foram realizadas por Conceição (2006) e são apresentadas no quadro 1.

As amostras de solo foram coletadas em minitrincheiras de $10 \times 40 \mathrm{~cm}$, secas ao ar, moídas e passadas em peneira de 2,0 $\mathrm{mm}$. Parte da amostra de solo foi analisada quanto ao teor de $\mathrm{C}$ orgânico por meio do método de combustão seca, em analisador Shimadzu TOC-V CSH, e a outra parte, submetida ao fracionamento densimétrico. As FL e FP foram separadas por densimetria (Golchin et al., 1994b), em soluções de $\mathrm{NaI}\left(\mathrm{d}=1,8 \mathrm{~kg} \mathrm{~L}^{-1}\right)$ e PTS $(\mathrm{d}=1,8,2,0$ e $\left.2,2 \mathrm{~kg} \mathrm{~L}{ }^{-1}\right)$. O pH da solução de $\mathrm{NaI}$ foi reduzido para 4,0 mediante adição de $\mathrm{HCl} 1,0 \mathrm{~mol} \mathrm{~L}^{-1}$, para ser semelhante ao $\mathrm{pH}$ inicial da solução de PTS e evitar a solubilização de MO.

As etapas do fracionamento densimétrico, realizado em triplicata, são apresentadas na figura 1. Como ilustrado nessa figura, $80 \mathrm{~mL}$ de solução de $\mathrm{NaI}$ ou PTS foram adicionados a $20 \mathrm{~g}$ de solo em tubo de centrífuga de $100 \mathrm{~mL}$. O tubo de centrífuga foi disposto em recipiente com água e gelo, e a suspensão, submetida à dispersão por ultra-som utilizando energia de $250 \mathrm{~J} \mathrm{~mL}^{-1}$ para o PVd e $450 \mathrm{~J} \mathrm{~mL}^{-1}$ para o LVd, as quais foram determinadas previamente para obtenção da dispersão total do solo em partículas primárias, sendo a temperatura da suspensão mantida sempre abaixo de $20^{\circ} \mathrm{C}$. Após a dispersão, a suspensão foi centrifugada a $2.000 \mathrm{~g}$ por $90 \mathrm{~min}$, e a FL, recuperada pela passagem do sobrenadante em filtro de fibra de vidro (Whatmann GF/C), o qual foi lavado com água destilada, para remoção do resíduo do sal. Os filtros com a FL foram secos em estufa a $60^{\circ} \mathrm{C}$ por 24 h e moídos em gral de ágata. As soluções de $\mathrm{NaI}$ e PTS foram recuperadas e recicladas, de acordo com procedimento descrito em Six et al. (1999).

A FP sedimentada no tubo foi passada em peneira de $53 \mu \mathrm{m}$ com auxílio de jatos de água, visando à quantificação da MO particulada (MOP) não separada no fracionamento densimétrico. $\mathrm{O}$ material retido na peneira, composto de areia e MOP, foi transferido para potes de $100 \mathrm{~mL}$ e seco em estufa a $60^{\circ} \mathrm{C}$, sendo essa fração denominada de MOP residual. Para 
avaliar a contaminação da FL com $\mathrm{C}$ proveniente da fração argila, foi efetuada análise de Fe total $\left(\mathrm{Fe}_{t}\right)$ (Fey \& Dixon, 1983) na FL e na fração argila, obtida por meio de separação granulométrica (Quadro 1), segundo procedimento descrito em Gee \& Bauder (1986). A FL e a MOP residual foram pesadas e moídas, sendo estas frações e a fração argila analisadas quanto aos teores de $\mathrm{C}$, pelo método da combustão seca, em analisador Shimadzu TOC-V CSH. A concentração de $\mathrm{C}$ na FP foi obtida por diferença entre o $\mathrm{C}$ do solo integral e o C da FL (Conceição, 2006). Dessa forma, o C da MOP residual, embora quantificado separadamente, foi contabilizado como pertencente à FP.

A FL e a MOP residual foram caracterizadas qualitativamente com auxílio de microscopia óptica, tendo sido utilizado aumento de oito vezes, e o material foi fotografado com câmera digital. Para isso, $10 \mathrm{~mL}$ de uma suspensão de água e as respectivas frações foram filtrados em filtro de fibra de vidro e, posteriormente, submetidos à microscopia. As características estruturais da FL e da MOP residual obtida com as diferentes soluções foram avaliadas por RMN ${ }^{13} \mathrm{C}$ em espectrômetro Varian, modelo Unity 40, operando numa freqüência de radiação de $100,58 \mathrm{MHz}$ e tempo de contato de $1 \mathrm{~ms}$, com a polarização cruzada com ângulo mágico de rotação (CP/MAS). As análises de $\mathrm{RMN}{ }^{13} \mathrm{C} \mathrm{CP} / \mathrm{MAS}$, devido a seu alto custo, foram realizadas em amostras compostas formadas a partir das três repetições do fracionamento da matéria orgânica em laboratório. $\mathrm{O}$ tempo de aquisição e relaxação foi de $500 \mathrm{~ms}$, sendo aplicado alargamento de linha entre 50 e $100 \mathrm{~Hz}$. O espectro foi dividido em quatro bandas, que correspondem a diferentes tipos de C: 0-45 Hz MHz${ }^{-1}$ (C-alquil), 45-110 Hz MHz${ }^{-1}$ (C-O-alquil), 110-160 $\mathrm{Hz} \mathrm{MHz}^{-1}$ (C aromático), e 160-220 Hz MHz ${ }^{-1}$ (C carbonila) e a quantificação de cada tipo de $\mathrm{C}$ foi feita através da integração das áreas de seus respectivos picos no espectro. A MOP residual

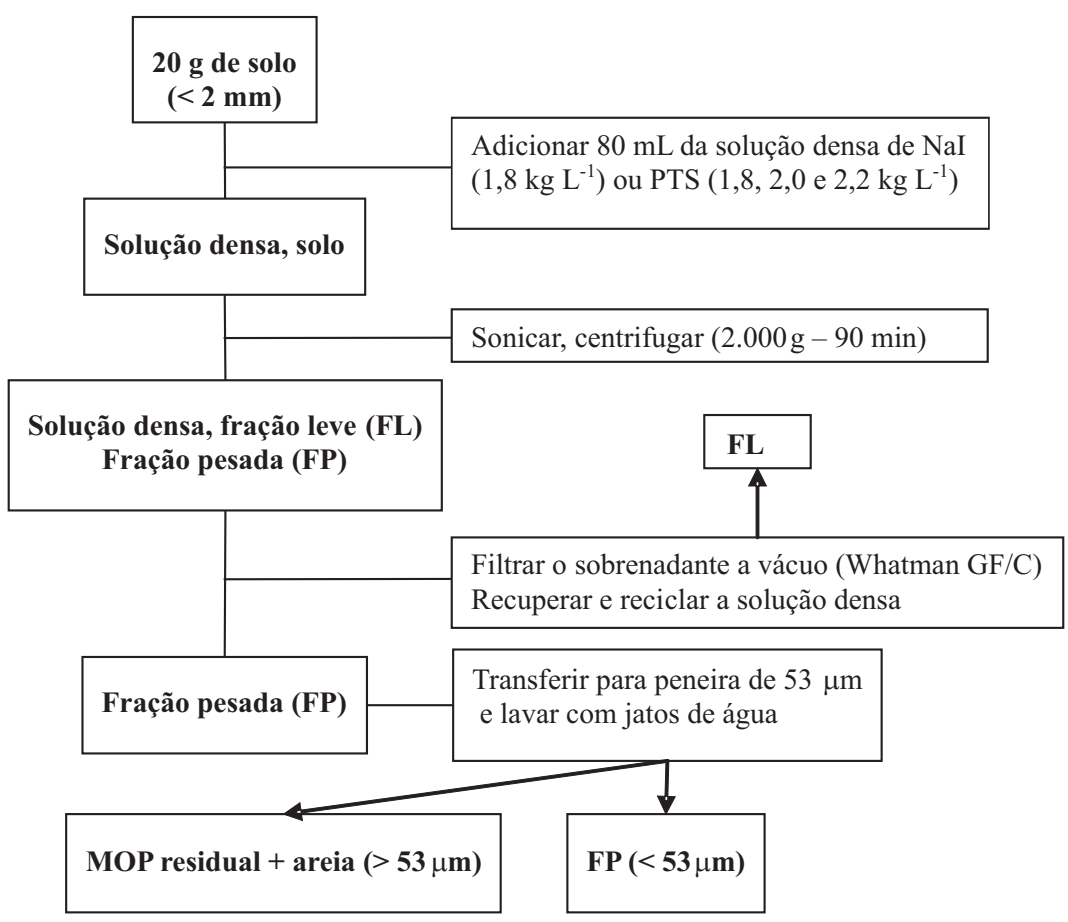

Figura 1. Organograma do fracionamento físico densimétrico. MOP: matéria orgânica particulada.

Quadro 1. Distribuição granulométrica e teores de ferro e de C orgânico na fração argila dos solos

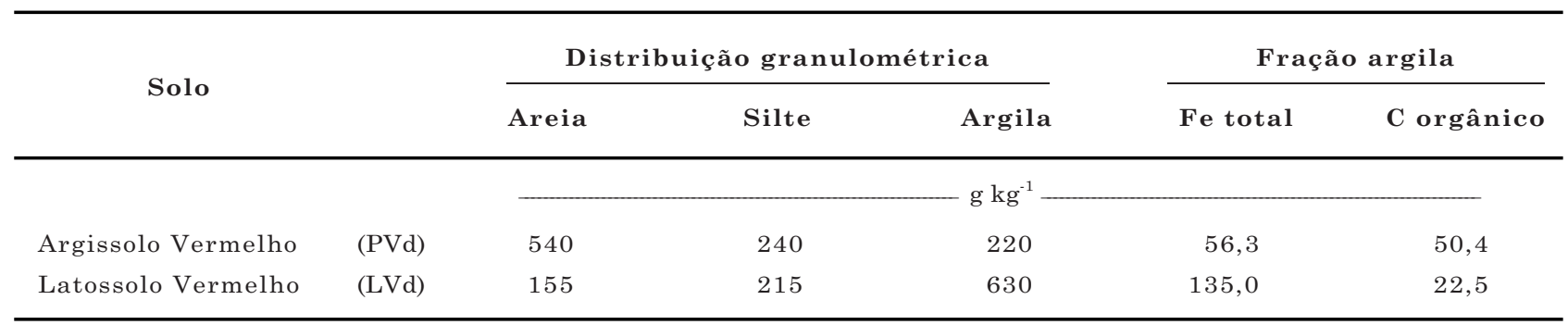

Fonte: Conceição (2006). 
somente foi analisada por $\mathrm{RMN}-{ }^{13} \mathrm{C}$ nos tratamentos com solução de NaI e PTS, ambas com densidade de $1,8 \mathrm{~kg} \mathrm{~L}^{-1}$, que apresentaram quantidade de material suficiente.

Os resultados do fracionamento referentes ao rendimento de $\mathrm{C}$ nas $\mathrm{FL}, \mathrm{FP}$ e MOP residual em cada solo foram submetidos à análise da variância, considerando um delineamento inteiramente casualizado, sendo as diferenças entre médias avaliadas pelo teste de Tukey a $5 \%$. Os resultados de RMN $-{ }^{13} \mathrm{C}$, obtidos de amostras compostas das três repetições do fracionamento em laboratório, não foram analisados estatisticamente.

\section{RESULTADOS E DISCUSSÃO}

A utilização da solução de PTS aumentou o rendimento de C na FL em 152 \% no PVd e em $166 \%$ no LVd, em comparação à solução de NaI, ambas com a densidade de 1,8 $\mathrm{kg} \mathrm{L}^{-1}$ (Quadro 2 e Figura 2a). Uma possível explicação para a menor recuperação da FL pelo uso de solução de $\mathrm{NaI}$ pode ser a formação de complexos da MO com o íon I-, conforme verificado por Radlinger \& Heumann (2000) em análises de complexação de compostos iodados com substâncias húmicas. Essa formação de complexos aumenta a densidade da FL e, conseqüentemente, diminui a recuperação dessa fração.

Além de proporcionar aumento do rendimento de $\mathrm{C}$ na FL, a utilização da solução de PTS $1,8 \mathrm{~kg} \mathrm{~L}^{-1}$ resultou na diminuição do $\mathrm{C}$ da MOP residual em $73 \%$ no PVd e 42 \% no LVd, demonstrando eficiência maior do PTS em relação ao NaI na recuperação da FL da MO do solo (Quadro 2 e Figura 2b). De fato, em grande parte a MOP residual deveria ter sido separada pelas soluções utilizadas no fracionamento densimétrico, e a diminuição da quantidade de $\mathrm{C}$ nesta fração indica aumento da eficiência da solução em separar a FL da MO do solo. A similaridade das estruturas orgânicas presentes na MOP residual e na FL é corroborada pela análise de microscopia óptica, que demonstrou que ambas as frações são constituídas basicamente de fragmentos de raízes e organismos da fauna do solo, hifas e resíduos de plantas em decomposição (Figura 3).

A baixa eficiência da solução de $\mathrm{NaI}$ pode ser reforçada a partir da reinterpretação dos resultados obtidos por outros pesquisadores em estudos de fracionamento densimétrico da $\mathrm{MO}$ do solo, nos quais tenha sido realizada a quantificação do $\mathrm{C}$ na fração areia (neste estudo denominado de MOP residual) posterior à separação da FL. Com a utilização de solução de NaI 1,8 $\mathrm{kg} \mathrm{L}^{-1}$ em Latossolos brasileiros (467 a $630 \mathrm{~g} \mathrm{~kg}^{-1}$ de argila), Freixo et al. (2002b,c) obtiveram valores de $\mathrm{C}$ na fração areia variando de 2 a 8,5 \% (0,32 a 2,39 $\mathrm{g} \mathrm{kg}^{-1}$ de C no solo) do C orgânico do solo; o $\mathrm{C}$ na fração areia representou $63 \%$ do $\mathrm{C}$ quantificado na FL (obtido pela soma do C da fração leve-livre e leve-oclusa). Roscoe et al. (2001), utilizando $\mathrm{NaI} 1,7 \mathrm{~kg} \mathrm{~L}^{-1}$, obtiveram teores de $\mathrm{C}$ na fração areia variando de 0,3 a $6,2 \mathrm{~g} \mathrm{~kg}^{-1}$ de solo, o que representou de 2,3 a $10,6 \%$ do $\mathrm{C}$ orgânico do solo, sendo o percentual de $\mathrm{C}$ recuperado na fração areia superior ao quantificado na FL e havendo tratamentos em que o C nessa fração chegou a ser quatro vezes maior do

Quadro 2. Recuperação de carbono na fração leve (FL), matéria orgânica particulada residual (MOP residual), fração pesada (FP) da matéria orgânica e concentração de ferro na FL obtidas com a utilização de soluções de iodeto de sódio (NaI) e de politungstato de sódio (PTS), em amostras da camada de 0-5 cm de um Argissolo Vermelho (PVd) e de um Latossolo Vermelho (LVd)

\begin{tabular}{|c|c|c|c|c|c|c|c|c|}
\hline \multirow{2}{*}{ Solo } & \multirow{2}{*}{ Produto } & \multirow{2}{*}{$\begin{array}{l}\text { Densidade } \\
\text { da solução }\end{array}$} & \multicolumn{2}{|c|}{ C na fração FL } & \multicolumn{3}{|c|}{ C no solo } & \multirow{2}{*}{ Fe na FL } \\
\hline & & & Quantidade & Teor & FL & MOP residual & $\mathbf{F P}$ & \\
\hline & & $\mathrm{kg} \mathrm{L}^{-1}$ & $\mathrm{~g}$ & 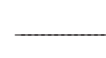 & $\mathrm{g}$ & $\mathrm{kg}^{-1}$ & $\underline{ }$ & $\mathrm{mg} \mathrm{g}^{-1}$ \\
\hline \multirow[t]{4}{*}{ PVd } & $\mathrm{NaI}$ & 1,8 & 0,12 & 30,27 & $1,83 \mathrm{~d}$ & $1,67 \mathrm{a}$ & 19,52 & 24,9 \\
\hline & PTS & 1,8 & 0,28 & 32,11 & $4,62 \mathrm{c}$ & $0,44 \mathrm{~b}$ & 16,74 & 24,3 \\
\hline & & 2,0 & 0,55 & 25,54 & $7,25 \mathrm{~b}$ & $0,22 \mathrm{~b}$ & 14,10 & 29,0 \\
\hline & & 2,2 & 0,91 & 24,34 & 8,76 a & $0,22 \mathrm{~b}$ & 12,60 & 32,0 \\
\hline \multirow[t]{4}{*}{$\mathrm{LVd}$} & $\mathrm{NaI}$ & 1,8 & 0,10 & 27,87 & $1,50 \mathrm{c}$ & $1,30 \mathrm{a}$ & 24,43 & 48,5 \\
\hline & PTS & 1,8 & 0,21 & 35,61 & $3,99 \mathrm{~b}$ & $0,75 \mathrm{~b}$ & 21,93 & 41,7 \\
\hline & & 2,0 & 0,38 & 30,33 & $6,31 \mathrm{a}$ & $0,44 \mathrm{bc}$ & 19,62 & 50,3 \\
\hline & & 2,2 & 0,57 & 23,17 & $6,64 \mathrm{a}$ & $0,40 \mathrm{c}$ & 19,29 & 49,3 \\
\hline
\end{tabular}

Valores seguidos de letras iguais na coluna, em cada solo, não diferem estatisticamente pelo teste de Tukey a $5 \%$. 
(a)

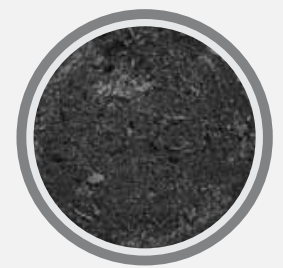

(b)

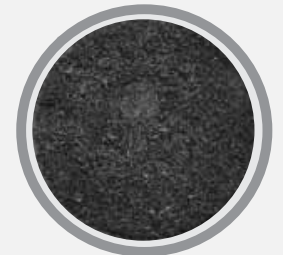

(a)

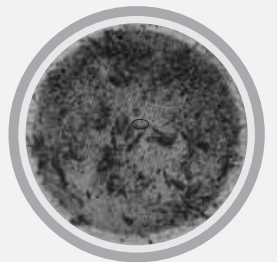

(b)

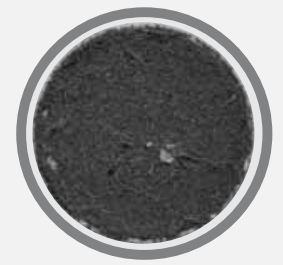

NaI $1,8 \mathrm{~kg} \mathrm{~L}^{-1}$
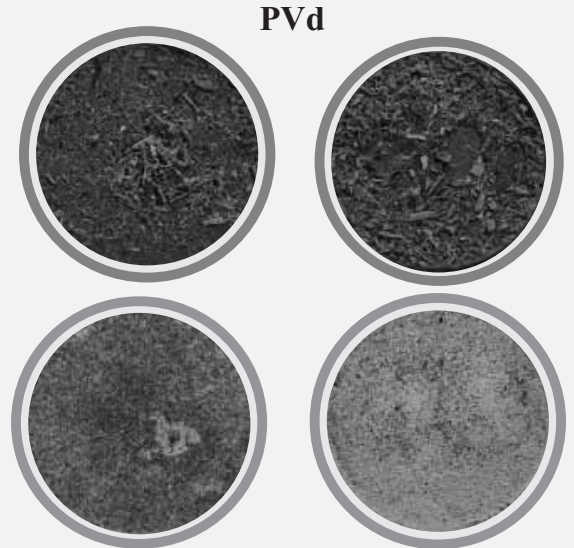

LVd
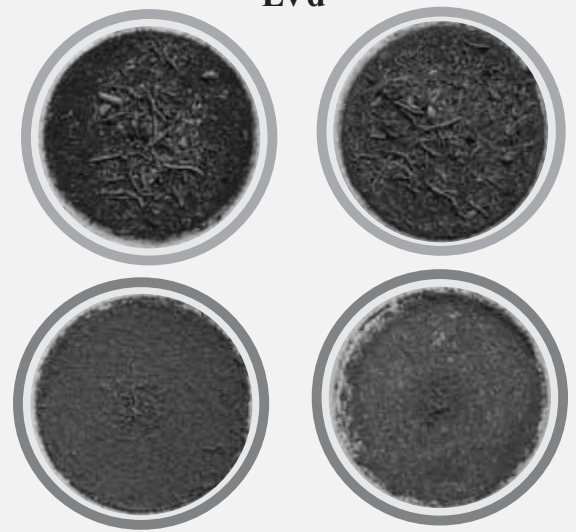

PTS $1,8 \mathrm{~kg} \mathrm{~L}^{-1}$
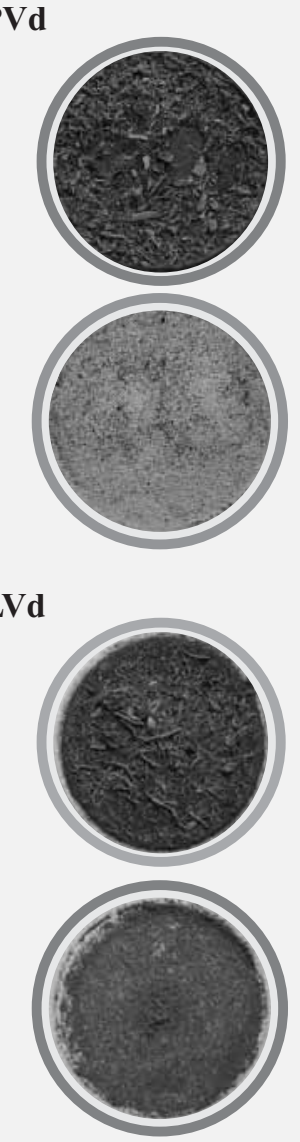

PTS 2,0 kg L $\mathrm{k}^{-1}$
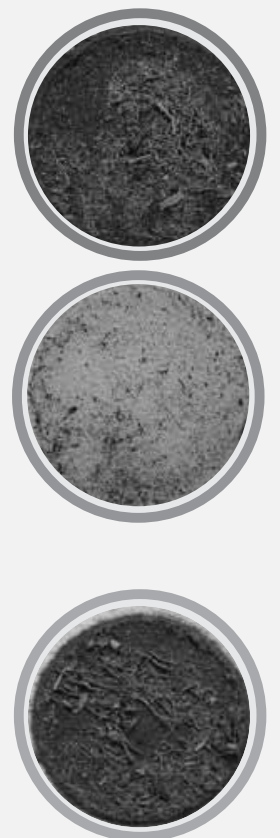

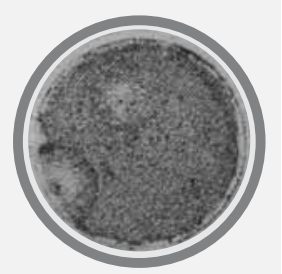

PTS 2,2 kg L

Figura 2. Visualização do aspecto dos filtros da fração leve (FL) (a) e da matéria orgânica particulada residual (b) utilizados no fracionamento densimétrico da matéria orgânica com soluções de iodeto de sódio (NaI) e de politungstato de sódio (PTS) na camada de 0-5 cm de um Argissolo Vermelho (PVd) e de um Latossolo Vermelho (LVd).

(a)

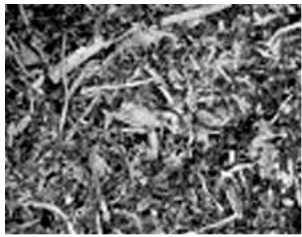

(b)

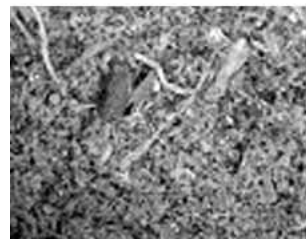

NaI 1,8 $\mathrm{kg} \mathrm{L}^{-1}$
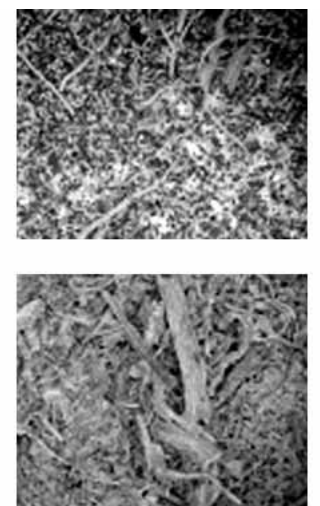

PTS $1,8 \mathrm{~kg} \mathrm{~L}^{-1}$
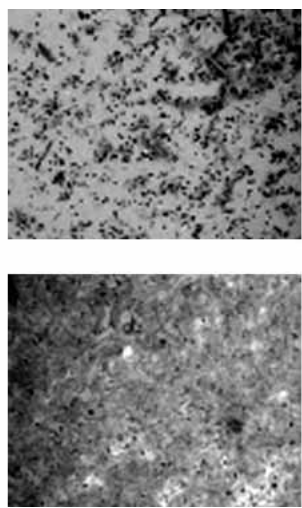

PTS 2,0 kg L $\mathrm{L}^{-1}$
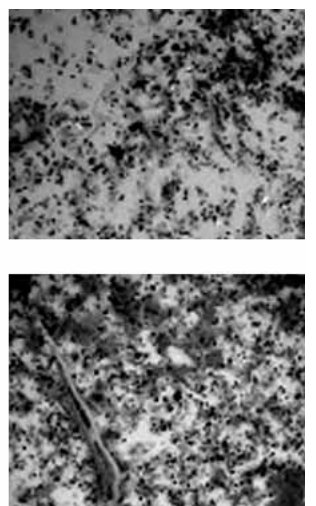

PTS 2,2 kg L $\mathrm{L}^{-1}$

Figura 3. Detalhe da matéria orgânica particulada residual obtida por peneiramento $(53 \mu \mathrm{m})$ da fração pesada após separação da fração leve com soluções de iodeto de sódio (NaI) e de politungstato de sódio (PTS), em um Argissolo Vermelho (a) e Latossolo Vermelho (b).

que o C na FL da MO do solo. Ou seja, a reinterpretação dos dados de fracionamento da literatura reforça a baixa eficiência da solução de $\mathrm{NaI}$ na recuperação da
FL da MO, a qual é ainda reduzida quando da utilização de soluções de menor densidade (Sohi et al., 2001). 
A recuperação de $\mathrm{C}$ na FL de ambos os solos no presente estudo aumentou em aproximadamente $57 \%$ com o aumento da densidade da solução de PTS de 1,8 para $2,0 \mathrm{~kg} \mathrm{~L}^{-1}$, sendo acompanhado por diminuição do $\mathrm{C}$ da MOP residual de $50 \%$ no PVd e de $41 \%$ no LVd. Assim, em comparação com a solução de NaI $1,8 \mathrm{~kg} \mathrm{~L}^{-1}$, houve diminuição na MOP residual de $87 \%$ no PVd e de 66 \% no LVd quando do uso da solução de PTS $2,0 \mathrm{~kg} \mathrm{~L}{ }^{-1}$. Por sua vez, o teor de $\mathrm{C}$ recuperado na fração MOP residual quando do uso da solução de PTS 2,0 kg L $\mathrm{kg}^{-1}$ representou aproximadamente $1 \% \mathrm{da}$ concentração do C orgânico em ambos os solos e apenas 3 e $7 \%$ do C quantificado como FL no PVd e LVd, respectivamente.

O subseqüente incremento na densidade da solução de PTS de 2,0 para $2,2 \mathrm{~kg} \mathrm{~L}^{-1}$ resultou em aumentos menos expressivos na recuperação de $\mathrm{C}$ na FL ( $21 \%$ no PVd e $5 \%$ no LVd), não ocorrendo diminuição significativa do $\mathrm{C}$ na fração MOP residual em ambos os solos. Além disso, o uso de solução de PTS $2,2 \mathrm{~kg} \mathrm{~L}^{-1}$ dificultou o manuseio da amostra no momento da filtragem da FL, devido à baixa consistência do "pellet" após a centrifugação.

Ao se calcular a diferença entre a concentração de $\mathrm{C}$ da FL obtida pelo uso de PTS nas densidades de 1,8 e 2,0 kg L $\mathrm{kg}^{-1}$ no PVd, obteve-se o valor absoluto de $2,63 \mathrm{~g} \mathrm{~kg}^{-1}$ de C no solo, enquanto o mesmo cálculo para o C da fração MOP residual resultou em $0,22 \mathrm{~g} \mathrm{~kg}^{-1} \mathrm{de}$ $\mathrm{C}$ no solo. Isso evidencia que apenas uma pequena parte ( 8 \%) da FL que não foi separada pela solução de PTS $1,8 \mathrm{~kg} \mathrm{~L}^{-1}$ foi recuperada pelo peneiramento $(53 \mu \mathrm{m})$ da FP e quantificada como MOP residual, e isso se deve ao fato de que o ultra-som resulta num fracionamento do tamanho de partícula da FL (Balesdent et al., 1991) e grande parte dessa fração não foi retida na peneira utilizada. Da mesma forma, o uso da solução de PTS $2,0 \mathrm{~kg} \mathrm{~L}^{-1}$ no LVd resultou em aumento de $2,34 \mathrm{~g} \mathrm{~kg}^{-1}$ de C no solo na FL e redução de apenas $0,31 \mathrm{~g} \mathrm{~kg}^{-1}$ de C no solo na fração MOP residual, em comparação à solução de PTS $1,8 \mathrm{~kg} \mathrm{~L}^{-1}$. Esses resultados indicam que a quantificação da MOP residual serve apenas para caracterizar as soluções quanto à sua eficiência na separação da FL da MO do solo, não representando uma alternativa adequada para ser usada complementarmente ao fracionamento densimétrico com solução de $\mathrm{NaI}$, bem como quando do uso de solução de PTS com baixa densidade.

$\mathrm{O}$ efeito das soluções de $\mathrm{NaI}$ e de PTS na quantificação do $\mathrm{C}$ na FL afetou os resultados também de C na FP, o qual foi calculado por diferença em relação aos estoques de C orgânico no solo. Dados de literatura, utilizando NaI, indicam o C na FP como predominante nos solos, representando mais de $80 \%$ do C orgânico do solo (Roscoe et al., 2001; Sohi et al., 2001; Freixo et al., 2002a,b,c; Pinheiro et al., 2004). No presente estudo, utilizando $\mathrm{NaI} 1,8 \mathrm{~kg} \mathrm{~L}^{-1}$, o C na FP correspondeu a 91 e 94 \% do C orgânico do PVd e LVd, respectivamente (Quadro 2). Contudo, com o uso de PTS 2,0 kg L-1, o C na FP passou a representar uma menor proporção do C orgânico do solo (66 \% no PVd e 76 \% no LVd). Dessa forma, a importância da FP no estoque de $\mathrm{C}$ orgânico do solo foi diminuída pela maior recuperação de $\mathrm{C}$ na FL com a utilização do PTS, sendo esse um aspecto importante na interpretação de resultados obtidos em diferentes solos, visando avaliar a sua capacidade de interação e estabilização da MO.

O aumento na recuperação de C na FL da MO do solo ( $\mathrm{g} \mathrm{kg}^{-1} \mathrm{de} \mathrm{C}$ no solo) foi acompanhado, de maneira geral, por acréscimo na concentração de $\mathrm{Fe}_{t}$ e diminuição de $\mathrm{C}$ na fração ( $\mathrm{g} \mathrm{kg}^{-1}$ de $\mathrm{C}$ na fração) (Quadro 2), sugerindo contaminação com fração mineral (óxidos de Fe e argilominerais). Utilizando a FL obtida com a solução de $\mathrm{NaI} 1,8 \mathrm{~kg} \mathrm{~L}^{-1}$ como referência do teor de $\mathrm{Fe}_{\mathrm{t}}$, estimou-se que, com o uso da solução de PTS 1,8 kg L-1 não houve contaminação adicional com fração argila devido aos valores similares ou um pouco inferiores de $\mathrm{Fe}_{t}$ na FL obtida com essa solução. Por outro lado, o aumento de 16 e $28 \%$ na concentração de $\mathrm{Fe}_{\mathrm{t}}$ da FL obtida com as soluções de PTS 2,0 e $2,2 \mathrm{~kg} \mathrm{~L}^{-1}$ indicou provável contaminação com argila. Com base nos teores de $\mathrm{Fe}_{t}$ e de $\mathrm{C}$ orgânico da fração argila (Quadro 1), estimou-se que a contaminação da FL com C da fração argila variou de 6,8 a $10,4 \%$ no PVd e de 1,9 a $3,5 \%$ no LVd, a qual foi considerada baixa em todos os tratamentos, principalmente considerando o grande aumento verificado no rendimento de C na FL da MO em ambos os solos.

Os espectros de CPMAS-RMN ${ }^{13} \mathrm{C}$ apresentaram alta razão sinal/ruído em ambos os solos (Figura 4), tendo sido verificada melhor definição de bandas no $\mathrm{PVd}$, o que se deve à maior razão $\mathrm{C} / \mathrm{Fe}$ nas amostras do que no LVd (Quadro 2). A observação individual de bandas (Figuras 4 e 5) permite verificar tendência de aumento dos grupamentos C-carbonila $\left(160-220 \mathrm{~Hz} \mathrm{MHz}^{-1}\right)$ e de C-alquil $\left(0-45 \mathrm{~Hz} \mathrm{MHz}^{-1}\right) \mathrm{e}$ diminuição do C-O alquil $\left(45-110 \mathrm{~Hz} \mathrm{MHz}^{-1}\right)$ com o uso de solução de PTS em vez da solução NaI. O mesmo comportamento foi observado com o aumento da densidade da solução de PTS, sendo mais expressivo no LVd do que no PVd. Por sua vez, a razão C-alquil/ C-O alquil, a qual tem sido utilizada como um índice de humificação da MO (Dick et al., 2005), aumentou com o uso da solução de PTS, em comparação à solução de NaI (Figura 5), o que também se verificou com o aumento da densidade da solução de PTS, sendo esse efeito restrito ao LVd. A maior razão C-alquil/C-O alquil da MOP residual do que da FL, obtidas com soluções de NaI e PTS $1,8 \mathrm{~kg} \mathrm{~L}^{-1}$, no LVd também demonstra que a MO que não está sendo separada no fracionamento é mais humificada do que a FL obtida (Figura 5).

Todos esses indicadores obtidos da análise espectroscópica da FL demonstram que o uso de PTS e o aumento da densidade da solução promoveram maior recuperação de componentes em estádios mais avançados de decomposição da MO, em comparação à solução de NaI. Esses resultados são coerentes com 

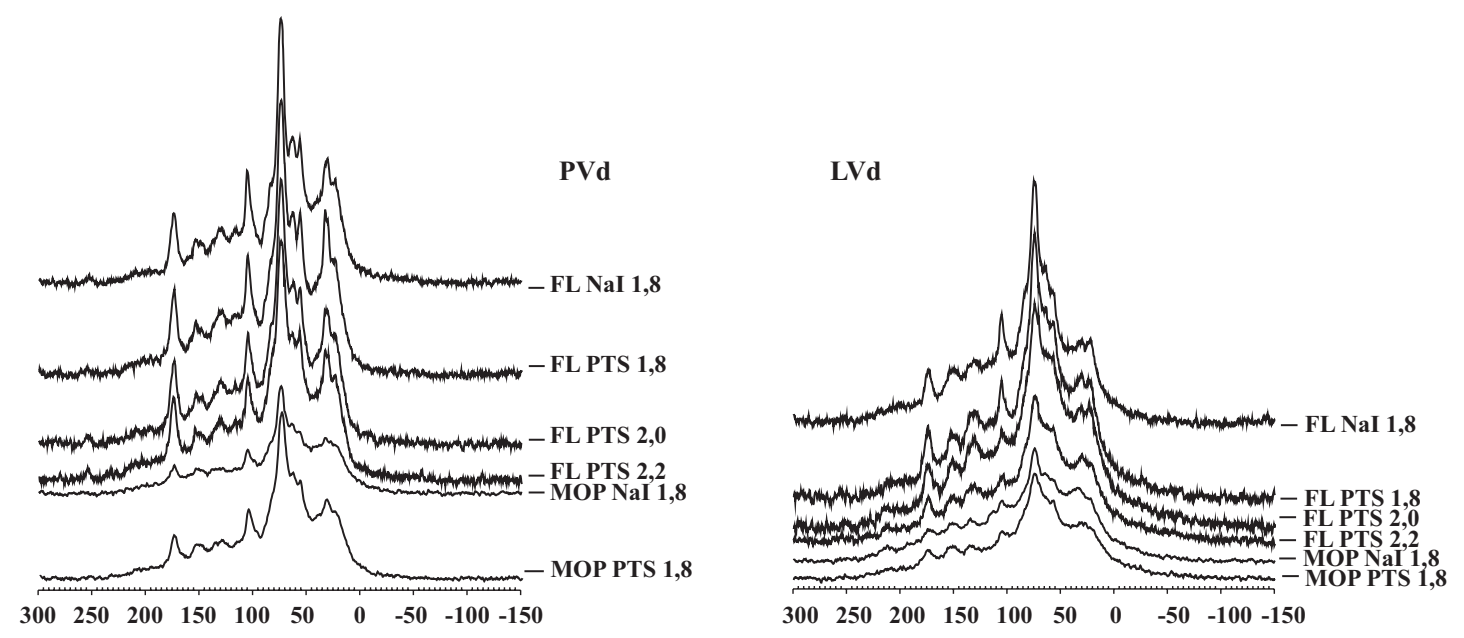

DESLOCAMENTO QUÍMICO, $\mathrm{Hz} \mathrm{MHz}^{-1}$

Figura 4. Espectros de $\mathrm{RMN}^{13} \mathrm{C}$ da fração leve (FL) e da matéria orgânica residual (MOP) de um Argissolo Vermelho (PVd) e de um Latossolo Vermelho (LVd) obtida em fracionamento densimétrico com soluções de iodeto de sódio (NaI) e de politungstato de sódio (PTS).

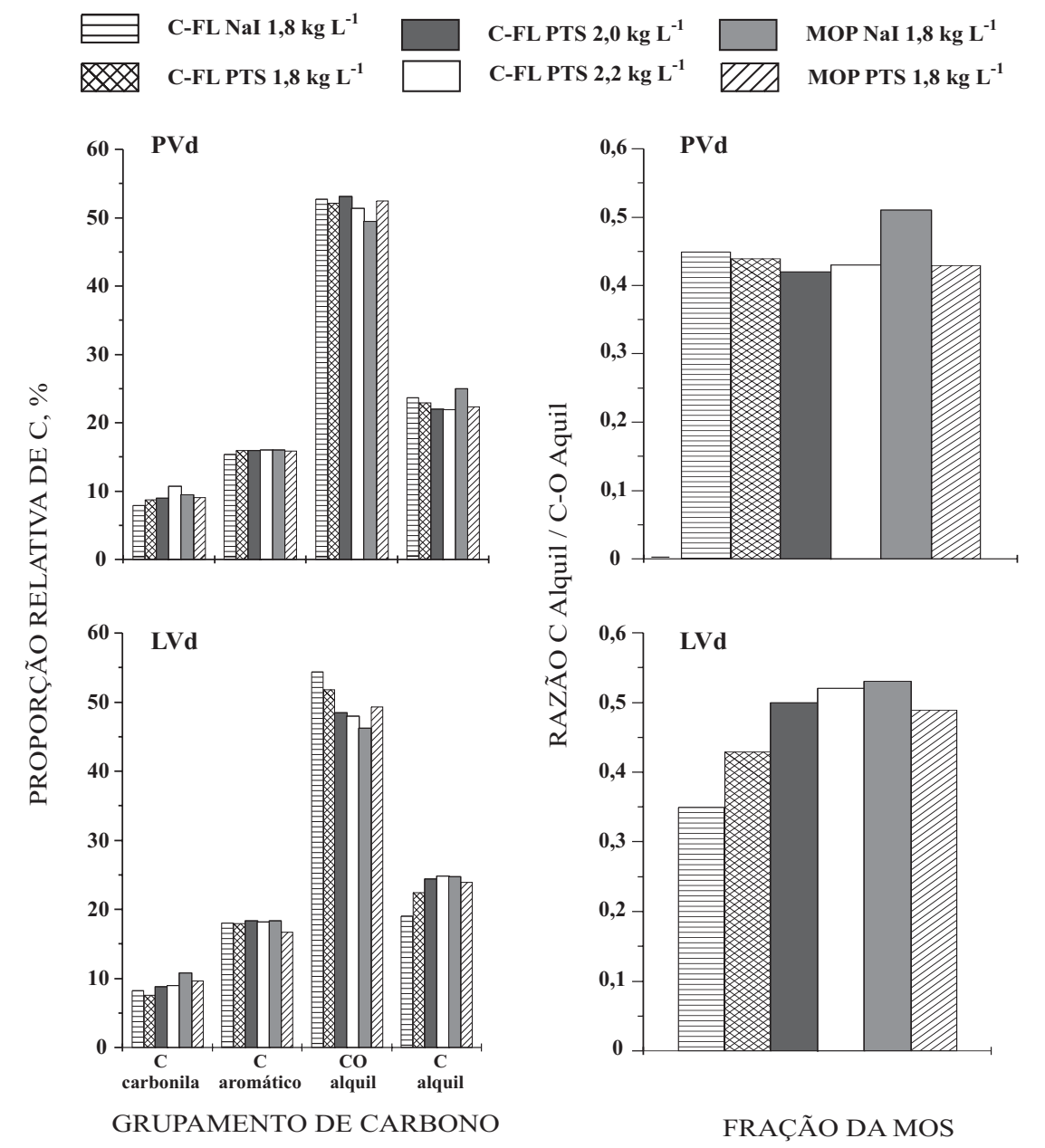

Figura 5. Distribuição relativa dos grupamentos de $\mathrm{C}$ quantificados por $\mathrm{RMN}{ }^{13} \mathrm{C}$ da fração leve da matéria orgânica (FL) e da matéria orgânica particulada residual (MOP) e razão entre os grupamentos C Alquil e C-O Alquil de um Argissolo Vermelho (PVd) e de um Latossolo Vermelho (LVd) obtida em fracionamento densimétrico com soluções de iodeto de sódio (NaI) e politungstato de sódio (PTS). Análises realizadas em amostras compostas das repetições de laboratório e não analisadas estatisticamente. 
os obtidos por Golchin et al. (1994a) e Sohi et al. (2001), que constataram incremento expressivo na recuperação da FL oclusa com o aumento da densidade da solução, não tendo sido observada alteração na recuperação da FL livre. A FL oclusa é uma fração que se encontra em estádio mais avançado de decomposição do que a FL livre, possuindo, portanto, menor proporção de carboidratos, que são os principais responsáveis pela intensidade do sinal relativo ao grupamento C-O Alquil.

\section{CONCLUSÕES}

1. A solução de politungstato de sódio é mais eficiente na recuperação da fração leve da matéria orgânica do solo do que a solução de iodeto de sódio.

2. O aumento da densidade da solução de politungstato de sódio determina acréscimo na recuperação da fração leve da matéria orgânica do solo, o qual é mais expressivo até a densidade de $2,0 \mathrm{~kg} \mathrm{~L}^{-1}$, e não se reflete em aumento expressivo da contaminação da fração leve por carbono da argila.

3. O uso do politungstato de sódio e de soluções com densidade maior que $1,8 \mathrm{~kg} \mathrm{~L}^{-1}$ resulta no aumento da razão C-alquil/C-O-alquil e do teor de C-carbonila na fração leve da MO, sugerindo sua maior eficiência na recuperação de compostos orgânicos em estádios mais avançados de decomposição.

4. A padronização do uso da solução de politungstato de sódio $2,0 \mathrm{~kg} \mathrm{~L}^{-1}$ é recomendada em estudos de fracionamento densimétrico da MO do solo.

\section{AGRADECIMENTOS}

À Fundação de Amparo à Pesquisa do Estado do Rio Grande do Sul, ao Conselho Nacional de Desenvolvimento Científico e Tecnológico (CNPq) e ao Ministério da Ciência e Tecnologia (MCT), pelo auxílio financeiro no âmbito do projeto PRONEX "Seqüestro de carbono e mitigação das emissões de gases de efeito estufa por sistemas conservacionistas de manejo e as oportunidades para o agronegócio no RS”. C. Bayer, L. Martin-Neto e J. Mielniczuk agradecem ao CNPq a bolsa de produtividade em pesquisa.

\section{LITERATURA CITADA}

BALESDENT, J.; PÉTRAUD, J.P. \& FELLER, C. Effets des ultrasons sur la distribution granulométrique des matières organiques des sols. Sci. Sol, 29:95-106, 1991.

BAYER, C.; MARTIN-NETO, L.; MIELNICZUK, J. \& CERETTA, C.A. Effect of no-till cropping systems on soil organic matter in a sandy clay loam Acrisol from Southern Brazil monitored by electron spin resonance and nuclear magnetic resonance. Soil Till. Res., 53:95-104, 2000.
BAYER, C.; MARTIN-NETO, L.; MIELNICZUK, J.; PILLON, C. N. \& SANGOI, L. Changes in soil organic matter fractions under subtropical No-Till cropping systems. Soil Sci. Soc. Am. J., 65:1473-1478, 2001.

CAMBARDELLA, C.A. \& ELLIOTT, E.T. Particulate soil organic-matter changes across a grassland cultivation sequence. Soil Sci. Soc. Am. J., 56:777-783, 1992.

CHRISTENSEN, B.T. Physical fractionation of soil and organic matter in primary particle size and density separates. Adv. Soil Sci., 20:1-90, 1992.

CHRISTENSEN, B.T. Physical fractionation of soil and structural and functional complexity in organic matter turnover. Eur. J. Soil Sci., 52:345-353, 2001.

CONCEIÇÃO, P.C. Proteção física da matéria orgânica em solos do Sul do Brasil. Porto Alegre, Universidade Federal do Rio Grande do Sul, 2006. 145p.(Tese de Doutorado)

CONCEIÇÃO, P.C.; AMADO, T.J.C.; MIELNICZUK, J. \& SPAGNOLLO, E. Qualidade do solo em sistemas de manejo avaliada pela dinâmica da matéria orgânica e atributos relacionados. R. Bras. Ci. Solo, 29:777-788, 2005.

DICK, D.P.; NUNES GONCALVES, C.; DALMOLIN, R.S.D.; KNICKER, H.; KLAMT, E.; KOGEL-KNABNER, I.; SIMOES, M.L. \& MARTIN-NETO, L. Characteristics of soil organic matter of different Brazilian Ferralsols under native vegetation as a function of soil depth. Geoderma, 124:319-333, 2005.

DIEKOW, J.; MIELNICZUK, J.; KNICKER, H.; BAYER, C.; DICK, D.P. \& KOGEL-KNABNER, I. Carbon and nitrogen stocks in physical fractions of a subtropical Acrisol as influenced by long-term no-till cropping systems and $\mathrm{N}$ fertilisation. Plant Soil, 268:319-328, 2005.

EMPRESA BRASILEIRA DE PESQUISA AGROPECUÁRIA EMBRAPA. Centro Nacional de Pesquisa de Solos. Sistema brasileiro de classificação de solos. Rio de Janeiro, 1999. 412p.

FELLER, C. \& BEARE, M.H. Physical control of soil organic matter dynamics in the tropics. Geoderma, 79:69-116, 1997.

FEY, M.U. \& DIXON, J.B. Rapid estimation of iron oxides in soils and clays by spectrophotometric analysis. Soil Sci. Soc. Am. J., 17:1261-1263, 1983.

FREIXO, A.A.; CANELLAS, L.P. \& MACHADO, P.L.O.A. Propriedades espectrais da matéria orgânica leve-livre e leve intra-agregados de dois Latossolos sob plantio direto e preparo convencional. R. Bras. Ci. Solo, 26:445-453, 2002a.

FREIXO, A.A.; MACHADO, P.L.O.A.; DOS SANTOS, H.P.; SILVA, C.A. \& FADIGAS, F.S. Estoques de carbono e nitrogênio e distribuição de frações orgânicas de Latossolo do cerrado sob diferentes sistemas de cultivo. R. Bras. Ci. Solo, 26:425-434, 2002b.

FREIXO, A.A.; MACHADO, P.L.O.A.; DOS SANTOS, H.P.; SILVA, C.A. \& FADIGAS, F.S. Soil organic carbon and fractions of a Rhodic Ferralsol under the influence of tillage and crop rotation systems in southern Brazil. Soil Till. Res., 64:221-230, 2002c. 
GEE, G.W. \& BAUDER, J.W. Particle-size analysis. In: KLUTE, A., ed. Methods of soil analysis - Part 1 Physical and mineralogical methods. Madison, Soil Science Society of America, 1986. p.383-411

GOLCHIN, A.; OADES, J.M.; SKJEMSTAD, J.O. \& CLARKE, P. Soil-structure and carbon cycling. Aust. J. Soil Res., 32:1043-1068, 1994a.

GOLCHIN, A.; OADES, J.M.; SKJEMSTAD, J.O. \& CLARKE, P. Study of free and occluded particulate organic-matter in soils by solid-state ${ }^{13} \mathrm{C}$ CP/MAS NMR-Spectroscopy and scanning electron-microscopy. Aust. J. Soil Res., 32:285$309,1994 b$.

HASSINK, J.; BOUWMAN, L.A.; ZWART, K.B.; BLOEM, J. \& BRUSSAARD, L. Relationships between soil texture, physical protection of organic matter, soil biota, and c and $\mathrm{n}$ mineralization in grassland soils. Geoderma, 57:105$128,1993$.

PINHEIRO, E.F.M.; PEREIRA, M.G. \& ANJOS, L.H.C. Fracionamento densimétrico da matéria orgânica do solo sob diferentes sistemas de manejo e cobertura vegetal em Paty do Alferes (RJ). R. Bras. Ci. Solo, 28:731-737, 2004.
RADLINGER, G. \& HEUMANN, K.G. Transformation of iodide in natural and wastewater systems by fixation on humic substances. Environ. Sci. Technol., 34:3932-3936, 2000.

ROSCOE, R.; BUURMAN, P.; van LAGEN, B. \& VELTHORST, E. Transformations in occluded light fraction organic matter in a clayey Oxisol; Evidence from ${ }^{13} \mathrm{C}$ CP MASNMR and delta ${ }^{13} \mathrm{C}$ signature. R. Bras. Ci. Solo, 28:811818,2004

ROSCOE, R.; BUURMAN, P.; VELTHORST, E.J. \& VASCONCELLOS, C.A. Soil organic matter dynamics in density and particle size fractions as revealed by the ${ }^{13} \mathrm{C} /$ ${ }^{12} \mathrm{C}$ isotopic ratio in a Cerrado's Oxisol. Geoderma, 104:185-202, 2001.

SHANG, C. \& TIESSEN, H. Sequential versus parallel density fractionation of silt-sized organomineral complexes of tropical soils using metatungstate. Soil Biol. Biochem., 33:259-262, 2001.

SIX, J.; SCHULTZ, P.A.; JASTROW, J.D. \& MERCKX, R. Recycling of sodium polytungstate used in soil organic matter studies. Soil Biol. Biochem., 31:1193-1196, 1999.

SOHI, S.P.; MAHIEU, N.; ARAH, J.R.M.; POWLSON, D.S.; MADARI, B. \& GAUNT, J.L. A procedure for isolating soil organic matter fractions suitable for modeling. Soil Sci. Soc. Am. J., 65:1121-1128, 2001. 